Wilcoxon rank-sum test for age at time of diagnosis. The natural logarithm of polA copies/uL was regressed on HIV status and polA geometric mean ratios (GMR) and corresponding 95\% confidence intervals $(95 \% \mathrm{CI})$ were generated.

Results Of 188 subjects with ES, 22 PLWH and 72 PWH had available polA results in blood or tissues. PLWH were more likely to be male $(84 \%$ vs $50.6 \%, \mathrm{p}=0.005)$. There were no differences in age (range 18-62) or ethnicity between PLWH and PWH. There were no differences in the proportion of patients with polA positivity between groups in blood $(72 \%$ vs $55 \%, \mathrm{p}=0.269)$, skin $(64 \%$ vs $57 \%, \mathrm{p}=0.763)$ or mucous membranes $(67 \%$ vs $67 \%, p=1.000)$. There were also no differences in the geometric means of polA copies in the blood $(\mathrm{GMR}=1.8,95 \% \mathrm{CI}$ 0.5-6.0, $\mathrm{p}$-value $=0.339)$, skin $(\mathrm{GMR}=2.8$, $95 \% \mathrm{CI} 0.4-21$, p-value $=0.307$ ) or mucous membrane samples (0.001, 95\%CI 0-27, $\mathrm{p}=0.284)$.

Conclusions HIV status was not associated with higher polA Tp burdens in blood or skin in subjects with early syphilis.

\section{P329 SCALE-UP OF A NOVEL TESTING SERVICE: EXAMINING HOW PUBLIC HEALTH POLICIES IMPACT THE CONTEXT FOR ONGOING PROGRAM IMPLEMENTATION}

\begin{abstract}
$1,2,3 \mathrm{H}$ Chang, ${ }^{4} \mathrm{~K}$ MacKinnon, ${ }^{1} \mathrm{D}$ Haag, ${ }^{5} \mathrm{~S}$ Bannar-Martin, ${ }^{6} \mathrm{M}$ Karlsson, ${ }^{7} \mathrm{C}$ Worthington, $1,2 \mathrm{M}$ Gilbert*, ${ }^{8} \mathrm{D}$ Grace. ${ }^{1} \mathrm{BC}$ Centre for Disease Control, Vancouver, Canada; ${ }^{2}$ University of British Columbia, Vancouver, Canada; ${ }^{3}$ CIHR Canadian HIV Trials Network, Vancouver, Canada; ${ }^{4}$ York University, Toronto, Canada; ${ }^{5} /$ sland Health Authority, Victoria, Canada; ${ }^{6}$ Interior Health Authority, Kelowna, Canada; 'University of Victoria, Victoria, Canada; ${ }^{8}$ University of Toronto, Toronto, Canada
\end{abstract}

\subsection{6/sextrans-2021-sti.382}

Background The role of macro-level contextual factors (e.g., healthcare system configuration, legislation, policy) in program implementation is well-documented, yet their changing function throughout all implementation phases is less established. We examined contextual factors shaping the ongoing implementation of GetCheckedOnline-an internet-based testing program for sexually transmitted and blood-borne infections (STBBIs) available since 2014 in British Columbia, Canada.

Methods An institutional ethnography approach was used to examine how the work processes of scaling-up the service were organized. Data were collected April 2019-February 2020 , including interviews $(n=25)$, meeting observations with implementers of GetCheckedOnline, and analyses of key documents (e.g., provincial policies, progress reports).

Results The provincial 2012 HIV framework emerged as central to the tasks of implementing sexual health services. We uncovered a disjuncture in implementers' continued task of extending the regional reach of innovative STBBI programming. On one hand, implementers actively sought regional scale-up, but on the other, their work was limited by the parameters set within HIV targeted funding and the service implementation challenges brought by underfunded comprehensive sexual health care provision. Between 2014-16, the HIV framework enabled implementers to identify the conceptual, financial, and operational means for program scale-up. The work of interdisciplinary teams formed for the rollout of the HIV framework supported further regional expansion in 2019. However, implementers' efforts to continue to expand STBBI testing services were limited by policy, fostering co- testing only when centred around HIV and having to administer tight, targeted funds.

Conclusion Our findings reveal that targeted policy premised on biomedical HIV exceptionalism paradoxically opens opportunities for scale-up of STBBI programming while closing other possibilities over the implementation cycle. This study underscores the need for policy frameworks not to remain static, draws attention to the ways in which policy can foreclose public health service availability, and encourages ongoing critical policy scrutiny to promote change.

\section{P331 ACCEPTABILITY OF ALTERNATIVE SEXUAL HEALTH SERVICE DELIVERY METHODS DURING THE COVID-19 PANDEMIC IN BRITISH COLUMBIA (BC), CANADA}

${ }^{1} \mathrm{~A}$ Ablona*${ }^{1}{ }^{1} \mathrm{H}$ Chang, ${ }^{2} \mathrm{~T}$ Salway, ${ }^{1,3} \mathrm{G}$ Ogilvie, ${ }^{1,3} \mathrm{~T}$ Grennan, ${ }^{1,3} \mathrm{~J}$ Wong, ${ }^{1} \mathrm{D}$ Haag, ${ }^{1} \mathrm{H}$ Pedersen, ${ }^{4} \mathrm{~S}$ Bannar-Martin, ${ }^{4} \mathrm{~L}$ Campeau, ${ }^{1} \mathrm{G}$ Ford, ${ }^{5} \mathrm{D}$ Grace, ${ }^{6} \mathrm{C}$ Worthington, ${ }^{1,3} \mathrm{M}$ Gilbert. ${ }^{1} B C$ Centre for Disease Control, Vancouver, Canada; ${ }^{2}$ Simon Fraser University, Burnaby, Canada; ${ }^{3}$ University of British Columbia, Vancouver, Canada; ${ }^{4}$ Vancouver Island Health Authority, Victoria, Canada; ${ }^{5}$ University of Toronto, Toronto, Canada; ${ }^{6}$ University of Victoria, Victoria, Canada

\subsection{6/sextrans-2021-sti.383}

Background In response to the COVID-19 pandemic, sexual health services have started to implement alternative service delivery methods that reduce in-person contact (e.g., telemedicine, virtual health). We sought to understand acceptability of alternative service delivery methods among sexual health service clients in BC, Canada.

Methods We used data from an online anonymous survey administered from 21/07/2020-04/08/2020 to clients (aged $\geq 16$ years) who had used the BC Centre for Disease Control's sexually-transmitted infection (STI) clinic and/or the GetCheckedOnline testing service in the year prior to COVID-19 public health responses (03/2020). We described participants' likelihood of using potential alternative sexual health service delivery methods, and conducted bivariate analysis to examine its association with experiencing any sexual health service access barriers during the pandemic.

Results Of the 1198 survey participants (aged 17-76 years), $48 \%$ identified as men, $47 \%$ as women, and $5 \%$ as another gender; $71 \%$ identified as White, $24 \%$ as racialized minorities, and $4 \%$ as Indigenous. Overall, support for using alternative STI testing models was high, with $88 \%$ likely to use at-home self-collection kits and $79 \%$ likely to use an express testing model (i.e., phone/video triage prior to specimen collection at a clinic). More participants were likely to discuss sexual health with a healthcare provider over the phone (64\%), compared with video visits (53\%) and text (49\%). Text messaging to receive STI test results and reminders were of high interest (71\% and 63\% likely to use, respectively). Likelihood of using alternative service delivery methods did not differ by participants' experience of access barriers, where $66 \%$ of total participants reported having avoided/delayed seeking services during 03/2020-07/2020.

Conclusion Likelihood of using potential alternative sexual health service delivery methods was high overall, including among participants who did not avoid/delay seeking services. Sustaining and expanding such services would facilitate access during and beyond the COVID-19 pandemic. 